\title{
Estudio y restauración de un casco griego del museo de Málaga
}

Constanza Rodríguez Segovia, Esther Núñez Pariente de León | Centro de Intervención del IAPH

URL de la contribución <www.iaph.es/revistaph/index.php/revistaph/article/view/3647>

\section{RESUMEN}

Durante el año 2012 se acometió una intervención arqueológica preventiva en un solar ubicado en las calles Jinete y Refino de Málaga, en la que, amén de importantes niveles medievales, se documentó una gran tumba en la cual había sido enterrado un individuo varón acompañado de un magnífico ajuar mortuorio. Este se componía, principalmente, por armamento adscribible a una panoplia hoplita (casco, punta de lanza y restos de un posible escudo), así como de otros elementos de carácter ritual y simbólico (escarabeo de cornalina engastado en oro suspendido por anillo de plata, pátera de plata con roseta central, par de varillas de plata y timaterio cerámico), que denotaban el alto nivel social del inhumado.

Una vez trasladados estos materiales al Taller de Patrimonio Arqueológico del Centro de Intervención del IAPH, se le han practicado todos aquellos estudios y análisis concluyentes en la ulterior actuación de restauración, que permitirá su adecuada conservación así como su uso social a través de su musealización.

En este artículo se recogen los datos que han aportado la investigación y tratamiento de una de las piezas más destacadas, el casco corintio.

\section{Palabras clave}

Arqueología | Cascos de armadura | Centro de Intervención | Edad del Hierro | Instituto Andaluz del Patrimonio Histórico | Intervención | Investigación | Metal | Yelmos | 


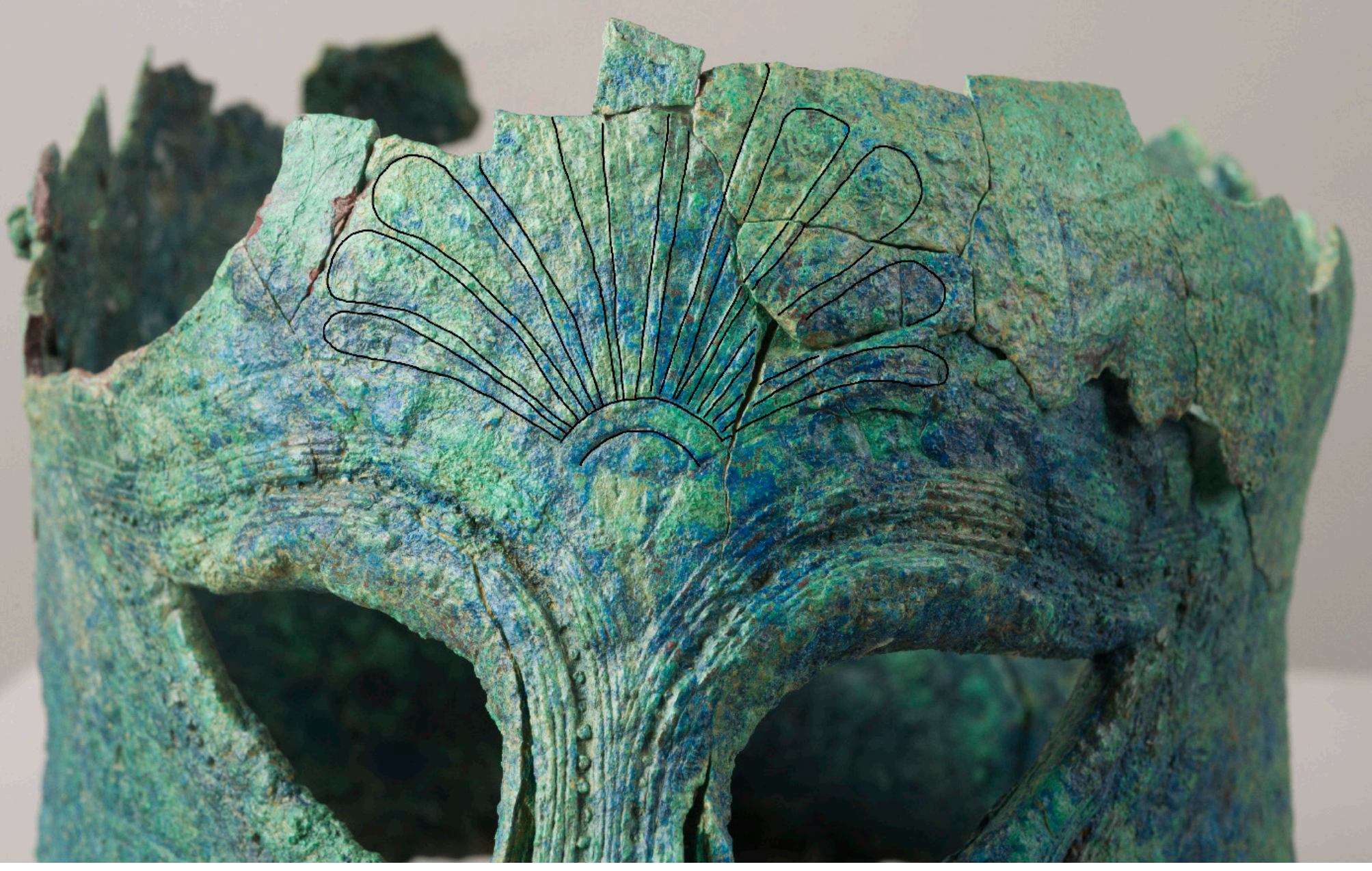

Frente con motivo de palmeta | foto Fondo Gráfico IAPH (Eugenio Fernández Ruiz); dibujo sobreimpreso Constanza Rodríguez Segovia 


\section{ESTUDIO HISTÓRICO-ARQUEOLÓGICO}

Este trabajo se desarrolló en el marco de la Encomienda de 19/09/2013, realizada por el museo de Málaga al IAPH.

\section{2}

En el s. VI a. de C., se pasa a adherir el forro al casco, por lo que las perforaciones ya resultan innecesarias; posteriormente (en torno al siglo $\mathrm{V}$ a. de $\mathrm{C}$.) se utilizan gorros, generalmente de fieltro, sobre los que se coloca el casco (GONZÁLEZ RODRÍGUEZ; BARRIONUEVO CONTRERAS; AGUILAR MOYA, 1997).

$$
3
$$

Respecto a las molestias que provocaba este tipo de cascos, ver Hanson, 1989: 73-74.

$$
4
$$

Estrabón, Nueve Libros de Historia, I, CLXXI: “... a la misma nación [carios] se debe el descubrimiento de tres cosas de que usan los griegos; pues ella fue la que enseñó a poner crestas o penachos en los morriones, a pintar armas y empresas en los escudos y a pegar en los mismos unas correas a manera de asas..."
En el transcurso de una excavación arqueológica realizada en el año 2012 en Málaga, dirigida por David García junto con Alberto Cumpián, Sonia López y Pedro J. Sánchez, fue localizado un enterramiento monumental del cual se recuperaron, además de los restos óseos (en muy malas condiciones) del difunto, extraordinarios materiales funerarios que fueron inmediatamente trasladados al museo de Málaga y, posteriormente, al Instituto Andaluz del Patrimonio Histórico para su restauración ${ }^{1}$. De entre los elementos hallados cabe destacar un casco griego.

Sin pretender profundizar a nivel de investigación arqueológica en este elemento, sí resulta necesario encuadrarlo cronotipológicamente de forma previa a desarrollar los resultados de la intervención física que se ha llevado a cabo sobre el mismo:

Se trata de un yelmo, del tipo denominado corintio, cuya funcionalidad consistía en dar protección a la mayor parte de la cabeza y zona posterior del cuello así como al rostro - gracias a la nariguera y las amplias carrillerasdejando sin cubrir exclusivamente los ojos y la parte inferior del mentón (como hándicap, ya que se perdía en gran medida la capacidad auditiva y la visión lateral). Como el material con el que se fabricaban -generalmente bronce o cobre- resultaba inapropiado (sobre todo por el calor) para llevar en contacto directo sobre piel y cuero cabelludo, se solían recubrir interiormente de fieltro, cuero o cualquier material orgánico similar; por otra parte, este forro amortiguaba parcialmente el efecto de la reverberación acústica en el caso de un golpe. En un principio, es decir, en los ejemplares más antiguos, se le realizaban pequeñas perforaciones simétricas que rodeaban todos sus filos, cosiéndose el forro, para mayor sujeción, a través de las mismas. En los casos mas evolucionados estas perforaciones desaparecen ${ }^{2}$, sustituyéndose generalmente la línea de puntos que representaban por motivos decorativos.

Respecto a su tipología, dentro de los escasos cambios formales que estos cascos sufrieron a lo largo de su vigencia como elementos defensivos, se observa que, inicialmente, eran prácticamente esférico-tubulares, sin mayor adaptación a la fisiología humana -lo que sin duda generaba molestias a su portador ${ }^{3}$, sobre todo en la zona de nuca y cuello-, evolucionando con el tiempo a una forma más ergonómica en la región posterior, hecho que revertiría en mayor comodidad para quien lo llevara; por otra parte, desde que los carios les enseñaron a los griegos el "modelo" con cimera o penacho (según recoge Estrabón $n^{4}$ ), este objeto decorativo y funcional fue utilizado habitualmente por los helenos, de tal manera que los remaches que hemos encontrado durante el proceso de extracción de su contenido podrían formar parte del sistema de fijación de una pieza de este tipo; así mismo, se aprecia un 
pequeño saliente en la zona medial trasera que tal vez sirvió para atarle alguna sujeción para colgarlo.

Por lo que a la cronología atañe, teniendo en consideración:

> que esta modalidad de yelmos presenta una gran amplitud tanto espacial (cuenca mediterránea mayoritariamente) como temporal (al menos desde la época arcaica hasta la clásica ${ }^{5}$ y, si nos remitimos a Homero ${ }^{6}$, incluso desde la edad oscura);

> que durante siglos se constata su utilización masiva por parte de los diferentes pueblos helénicos, pero también se han encontrado numerosos ejemplares en yacimientos fenicios, púnicos, etruscos e incluso en diferentes puntos de la Península Ibérica que, bajo estas circunstancias, podían pertenecer a individuos indígenas más o menos aculturizados por las corrientes venidas de oriente, a comerciantes fenicios o griegos. Montero Vítores (2002) defiende los intereses comerciales atenienses en las costas de Cádiz durante los siglos V y IV a. de C., argumentando las buenas relaciones que existían entre los focenses y Argantonio7;

> que la aparición de un elemento de estas características no tiene por qué obedecer a un contexto de guerras (siguiendo a Jiménez Ávila (2002) como en estas tierras no se dio ningún episodio bélico por esa época, los cascos, prácticamente, no tendrían función utilitaria), sino que pueden ser producto de comercio, intercambio, como manifestación de prestigio socioeconómico, con funciones votivas (los encontrados en Andalucía, recuperados del agua, fueron supuestamente arrojados a ella como parte de una ceremonia ritual), etc.; $>$ que según se conoce por diferentes fuentes cómo no todos los hoplitas tenían disponibilidad para adquirir una panoplia completa (según Jackson, 1991, tomado de GABALDÓN MARTÍNEZ, 2004: 122), en la Atenas de fines del s. VI a. de C., costaría unos 30 dracmas, igual que 6 bueyes de sacrificio) y, por tanto, en muchas ocasiones, ésta -total o parcialmente- se heredaba de padres a hijos;

$>$ y, finalmente, que también podría tratarse de armamento-trofeo (fruto de victorias en el combate) en cuyo caso alcanzaría una perdurabilidad aun más amplia.

Se concluye que no resulta fácil datar la pieza per se y sólo a partir de la contextualización arqueológica de la tumba y su ajuar al completo se podría ajustar su fechación.

No obstante, como datos específicos aplicables a este caso concreto, contamos con que, por un lado, morfoestilísticamente pertenece a una tipología ya evolucionada: si al casco cilíndrico, con perforaciones -más antiguo- aparecido en la desembocadura del río Guadalete (próximo a Jerez de la Frontera, Cádiz) en 1938, se le ha otorgado una cronología en torno al 630 a. de C. (SCHULTEN, 1979: 82 ) o 630-625 a. de C. (GARCÍA Y BELLIDO, 1948: 82 y ss.), al recu-
Tanto en la Anábasis de Jenofonte, como en la Anábasis de Alejandro Magno, de Lucio Flavio Arriano, se menciona tangencialmente la utilización de este tipo de casco, por lo que debió mantener alguna forma de pervivencia/ reutilización hasta época helenistica.

6

Así tanto en la llíada como en la Odisea, Homero hace numerosas alusiones a los cascos; en el caso de la primera, la somera descripción de los mismos nos revierte a la imagen de los prototipos corintios: “...reconozco su escudo, su casco de alta cimera y agujeros a guisa de ojos..." (Iliada, V-180); “...el fuerte Polipetes... hirió a Dámaso con la lanza por el casco de broncíneas carrilleras..." ( XI-182); etc.

$$
7
$$

Aunque parece sumamente improbable que en el siglo VI a. de C., y referido al sur peninsular, se diera ningún tipo de mercenariado -hecho que sí se constata a partir del siguiente siglo para zonas del centro-norte- (GRAELLS I FABREGAT, 2014), no se puede descartar que hubiera un cierto flujo de gentes de aquí -no con fines militares pero sí por intereses económicos- que a a su vuelta se trajeran los bienes materiales y culturales que hubieran adquirido durante su estancia en el ámbito helénico. 
Olmos, R. lo fecha a mitad del siglo VI a. de C. Igualmente lo datan en este siglo Albelda y Obermaier (ALBELDAALBERT; OBERMAIER, 1988) perado en la ría de Huelva en 1928, de factura más "moderna" (más similar al que estamos tratando), se le considera sobre el 550-525 a. de C. ${ }^{8}$ y al denominado etrusco-corintio (TIEMBLO MAGRO, 1994: 32-35; JIMÉNEZ ÁVILA, 2002: 237) posiblemente recuperado por la desembocadura del Guadalquivir (junto a Sanlúcar de Barrameda, Cádiz), de la mitad del s. VI a. de C., al que nos ocupa debemos encuadrarlo de forma genérica en el siglo $\mathrm{VI}$ a. de $\mathrm{C}$.

Respecto al vaciado de los sedimentos que colmataban el casco, realmente se ha efectuado como una microexcavación de su contenido. Se aplicó metodología arqueológica y rigor científico, separando en microniveles e individualizando los depósitos a medida que se iban extrayendo, al objeto de poder identificar, mediante su caracterización microestratigráfica, cualquier fragmento o resto de interés. De hecho, entre otros materiales se recuperaron del interior pequeños terrones con presencia orgánica que, una vez analizados, verifican la posible presencia de cuero, dato de gran importancia para determinar la existencia y naturaleza del previsible forro. También se tuvo en consideración la posición en la que se habían ido superponiendo los fragmentos correspondientes al propio casco, para intentar calibrar si este se había roto por la caída del techo de la tumba, o había sido fracturado de forma intencionada como parte del ritual funerario, coligiendo que, según parece, las roturas no se ocasionaron de manera exprofesa.

\section{EL CASCO GRIEGO Y SU SIGNIFICADO}

En la actualidad se cuenta con cerca de 2.000 ejemplares de cascos griegos que permiten llevar a cabo un estudio de su desarrollo histórico ya que muchos de ellos proceden de excavaciones y, por lo tanto, no están descontextualizados.

Entre el armamento de un guerrero griego, el casco alcanza un importante significado a la vez práctico y estético como se deduce de los abundantes ejemplos que encontramos en manifestaciones artísticas y literarias. Se trata de un componente relevante de la panoplia del hoplita ya que denotaba su estatus social y militar.

En la antigua Grecia los cascos podían constituir una ofrenda para un santuario o bien podían acompañar a su poseedor en la sepultura como es el caso del casco hallado en Málaga y de la mayor parte de las armas halladas en la Península Ibérica que se localizan preferentemente en necrópolis. Los enterramientos con armas fueron desapareciendo a lo largo del siglo VIII al tiempo que se incrementaba la presencia de depósitos de armas en los santuarios como es el caso de Delfos, Isthmia y Olimpia (Grecia) donde se exhibían colgados en la pared o bien formando parte de trofeos. De los más de 800 cascos hallados en Olimpia, actualmente se conservan más de 200 ejemplares de tipología corintia en su museo. 
Es frecuente que los cascos sufrieran una inutilización intencionada cuya finalidad sería evitar que pudieran ser reutilizados o bien convertirlos en piezas votivas. De este modo, armas que antes habían tenido su función práctica en el combate pasan a la esfera de lo simbólico.

La inutilización de un casco podía llevarse a cabo de diversas maneras: doblando los cubre mejillas hacia afuera, agujereándolos, aplastando la calota o escribiendo inscripciones en su superficie.

\section{ADSCRIPCIÓN TIPOLÓGICA}

Los cascos griegos se diferencian por su técnica de fabricación y por su forma. Las diversas tipologías (Kegel, Ilirio, Corintio, Calcídico, Ático, Frigio, Beocio y el tipo Pilos), así como sus numerosas variantes, reflejan la evolución que sufrieron para conseguir una mayor eficacia y ergonomía.

El casco protegía la cabeza del guerrero, exceptuando las aberturas correspondientes a los ojos y la boca. De todos ellos, el tipo de casco más extendido y popular fue, sin duda, el corintio que, según demuestra la evidencia cronológica, se comenzó a fabricar hacia el 700 y perduró hasta el s. V a. de C. constituyendo un importante logro a nivel técnico por estar fabricado en una sola lámina de bronce.

A lo largo de la primera mitad del s.VII a. de C. se convirtió en parte integral del armamento hoplita. Se dejó de fabricar porque impedía la buena visión y audición del guerrero. A pesar de la denominación que recibe por haberse hallado en Corinto una gran cantidad de este tipo de cascos y por haberse representado frecuentemente en vasos corintios, el adjetivo no determina su origen aunque sí identifica un modelo concreto fabricado en Grecia y la Magna Grecia del que, por otra parte, existen numerosas variantes.

De los 14 cascos recuperados hasta el momento en la Península Ibérica, el ejemplar hallado en Málaga es el único que no procede de contexto fluvial. El hecho de haberse encontrado el resto en este ámbito constituye una peculiaridad y ha llevado a su interpretación como ofrendas a divinidades fluviales (OLMOS, 1992).

De Andalucía proceden, incluyendo el casco objeto de este artículo, un total de 4 ejemplares de esta tipología datados entre mediados del s.VII y el s. $\mathrm{VI}$ a. de C. Se trata del casco griego arcaico del s. VII a. de C. hallado en el río Guadalete que se conserva en el Museo Arqueológico de Jerez; el casco griego de tipo corintio de la Ría de Huelva del s. VI a. de C. custodiado en la Real Academia de la Historia y el casco corintio tardío de mediados del 
s. VI a. de C. hallado en la desembocadura del Guadalquivir (Sanlúcar de Barrameda, Cádiz) y de propiedad particular.

En el ejemplar de Málaga la parte trasera de la nuca (guardanucas) tiene una curvatura acentuada. Tanto las escotaduras laterales de forma triangular que facilitaban su manipulación como la curvatura que presenta en su borde inferior son características de un momento avanzado en la evolución en este tipo de cascos. Sus rasgos tipológicos lo encuadran en el tipo II de la clasificación creada por Pflug (1988).

Tendría cimera como se desprende de los remaches $(17 \times 7 \mathrm{~mm})$ conservados en la calota que, como se verá más adelante, pudo ser reconstruida parcialmente durante el proceso de restauración. Sin esta última las dimensiones de la pieza son: altura: $21 \mathrm{~cm}$; anchura: $23 \mathrm{~cm}$; profundidad: $29 \mathrm{~cm}$. Con sus fragmentos reconstruidos y situándola sobre el resto de la pieza serían aproximadamente $29 \mathrm{~cm}$ de alto, $23 \mathrm{~cm}$ de ancho y una profundidad de $31 \mathrm{~cm}$. El peso total de las dos partes conservadas es de $2.246,4 \mathrm{~g}$ correspondiendo $1.737,3 \mathrm{~g}$ al casco sin calota y $509,1 \mathrm{~g}$ a esta última.

\section{TÉCNICA Y DECORACIÓN}

La lliada de Homero es la primera fuente escrita en la que se recogen aspectos tecnológicos y se describe la apariencia que tendrían los cascos en el mundo helenístico haciéndose mención a su brillo. Por su parte, las representaciones en la cerámica griega de figuras rojas aportan abundante información sobre las técnicas y el instrumental empleado en su fabricación.

Tal como se desprende de los ejemplares hallados hasta el momento durante el periodo geométrico tardío, los cascos griegos serían de cobre mientras que hacia el $700 \mathrm{a}$. de C. se generaliza el empleo del bronce en su fabricación. De hecho, la aleación y el sistema de fabricación tenían una gran importancia en el aspecto final del casco por lo que los fabricantes seleccionaban los metales y sus aleaciones no sólo por sus propiedades sino también por su color natural (HUGHES, 1993) y sus cualidades estéticas.

Probablemente se realizarían sobre un yunque como se puede constatar en el Kylix de figuras rojas de Orvieto del Ashmolean Museum (Oxford) o en una de las manifestaciones artísticas más antiguas sobre la fabricación de cascos corintios: la estatuilla del Metropolitan Museum of Art de Nueva York con n. ${ }^{\circ}$ de inventario 42.11 .42$.

Entre las herramientas empleadas se pueden mencionar los buriles, punzones, escofinas, martillos de diferentes formas y tamaños, tenazas, etc. Se empleaba la combinación de la fundición y el martilleado de la lámina 
de bronce con lo que se reducía el peso y se conseguía un metal de gran dureza, acorde con la función protectora de la pieza. Mediante el empleo de distintos tipos de martillos, el fabricante ajustaba el trabajo a las necesidades de cada una de las fases del mismo. En algunos cascos se han podido detectar las huellas del martillo sobre la superficie metálica (MANTI, 2012). Posteriormente el recocido permitía devolver al metal las condiciones necesarias para poder seguir dándole la forma deseada lo cual, al mismo tiempo, era intencionado puesto que se aportaba una mayor resistencia a zonas como el protector nasal, la frente o las mejillas.

Esta técnica de fabricación daba lugar a que la lámina de bronce alcanzara un grosor variable según la zona del casco siendo las más gruesas el protector nasal, la zona inferior y el borde. En el casco de Málaga el protector nasal tiene un grosor máximo de $18 \mathrm{~mm}$.

En la calota, sin embargo, frente a otros ejemplares en los que el grosor puede llegar a $1.5 \mathrm{~mm}$ se alcanzan los $0.02 \mathrm{~mm}$. El escaso grosor en esta zona viene dado por la técnica del batido a la que sería sometido.

Los cascos solían llevar un forro en su interior realizado en diversos materiales orgánicos cuya finalidad era aislar el metal y amortiguar los golpes. Podían ser de cuero e irían fijados con remaches a través de pequeños orificios o perforaciones que recorrían el borde a lo largo del perímetro de los ojos y en la zona inferior como en el casco del museo de Málaga o mediante algún tipo de encolado del que no han llegado restos.

Con la misma finalidad podían recurrir también a llevar un gorro de fieltro o a ceñirse una banda de tela ancha en la cabeza (QUESADA SANZ; VALERO TÉVAR, 2011-12).

La decoración en los cascos se sitúa en los cubre-mejillas, el frontal o el cubre-nuca. Las técnicas más frecuentes son el grabado y el repujado aunque hay evidencias de ejemplares con restos de dorado, plateado y estañado (acabado), patinación artificial o policromía (MANTI; WATKINSON, 2008).

La aplicación de dorado, que se menciona en la Iliada haciendo referencia a la decoración de escudos y a la cresta de un casco, se ha visto confirmada con el hallazgo en 2012 de un casco corintio en Haifa (Israel) que presentaba restos de dorado. La presencia de remaches plateados se ha estudiado en varios casos como en el casco 15153 del Museo Arqueológico Nacional de Atenas de mediados del siglo VI a. de C. y en un ejemplar del museo de Olimpia, entre otros.

La aplicación de una fina lámina de plata sobre el bronce se podía aplicar, según los estudios realizados, mediante soldadura de estaño o adhiriéndola 


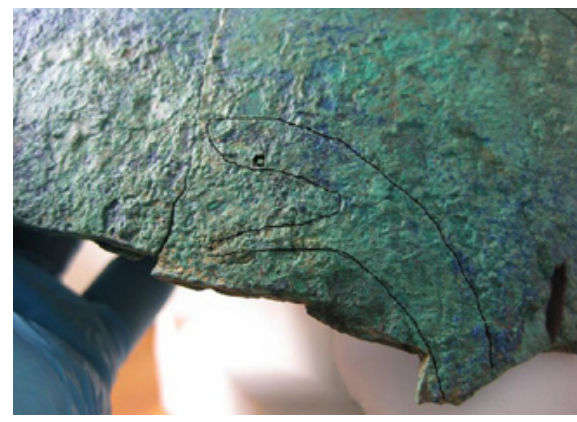

Motivo de serpiente en lateral izquierdo

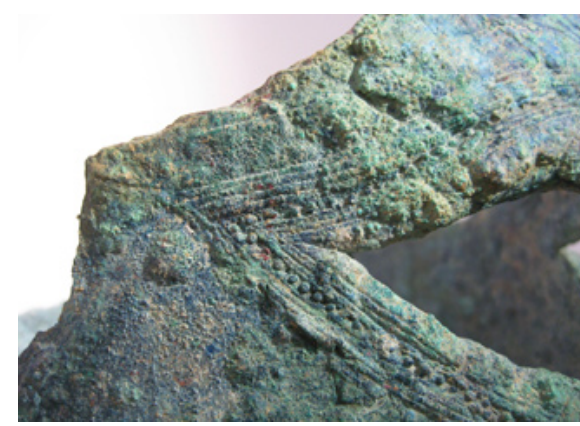

Franjas decorativas en el contorno del ojo derecho

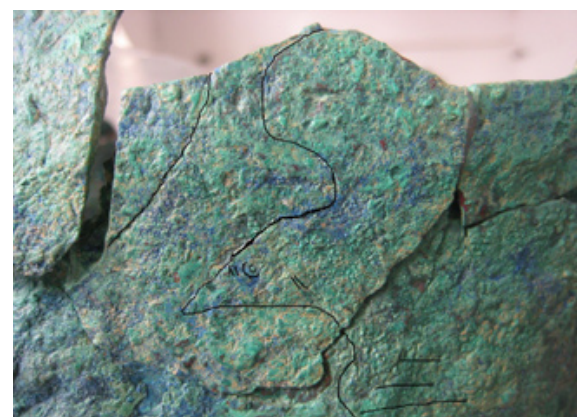

Águila superior en lateral izquierdo

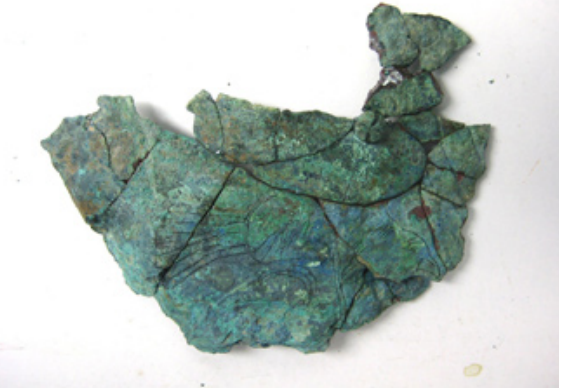

Fragmentos reconstruidos en zona superior del lateral derecho | fotos Fondo Gráfico IAPH (Constanza Rodríguez Segovia) con un adhesivo con base de calcita. Por lo que se refiere al estañado, el casco Gr 1856.12-26.616 del museo Británico, del s. V a. de C., se menciona como el ejemplo más antiguo de objeto estañado en el Mediterráneo (MANTI, 2012).

Uno de los mejores ejemplos de decoración grabada es el casco hallado en la isla de Giglio (Italia) en 1962. Está decorado con serpientes sobre las cejas, palmeta en el frontal y jabalíes en los cubre-mejillas.

En el casco de Málaga se encuentra, así mismo, el motivo de la palmeta y el de las serpientes que se extienden por encima de las aberturas de los ojos volviéndose hacia el interior de tal manera que sus cabezas se contraponen a ambos lados de esta última.

Sobre la punta de la escotadura lateral izquierda se puede ver una palmeta de loto en la que los pétalos divergentes arrancan de dos espirales. En el otro lado tendría la misma decoración pero el avanzado estado de corrosión impide su correcta visualización.

El borde que rodea todo el contorno inferior, el contorno de los ojos y el protector nasal cuenta con dos franjas de tres líneas incisas en ambos casos entre las que discurren pequeños orificios. En estos últimos se introdujeron clavitos de cabeza redondeada.

Se ha podido estudiar la existencia de algunos clavos en los que la parte del vástago que sobresalía por el interior fue golpeada para doblarlos evitando así que se desprendieran. Es posible que sirvieran para unir el gorro que amortiguaría los golpes.

Los cubre-mejillas, por su parte, están decorados con motivos de águilas, tan frecuentes en otros soportes como la cerámica.

\section{ESTUDIOS CIENTÍFICOS}

La conservación, a través de los tratamientos que han permitido devolver a la pieza su estabilidad y su lectura, y el estudio, mediante el que se ha buscado conseguir la mayor cantidad de información posible a lo largo de las distintas fases del trabajo, han constituido los principales objetivos de la intervención.

Desde el primer proceso, la radiografía, se ha tenido la oportunidad de conocer aspectos relevantes sobre la pieza referentes tanto a su estado de conservación como a su decoración.

Las radiografías se realizaron una vez vaciado el casco de la tierra que contenía. Permitieron detectar la existencia de diferentes tipos de decoración en 
la pieza. Se podían observar líneas incisas que parecían corresponder a un ave en los cubre-mejillas, una palmeta sobre la escotadura lateral derecha y lo que parecían pequeños orificios decorativos en todo el contorno del borde, el perímetro de los ojos y el protector nasal.

El estudio analítico realizado se ha centrado en la identificación de los materiales presentes en el casco. Para conocer la composición química del metal se ha sometido la pieza a un estudio arqueometalúrgico por parte de Mark Hunt Ortiz quien ha contado con las analíticas proporcionadas por el Centro Nacional de Aceleradores (CNA) y el Laboratorio de Arqueometría de Metales (LAM) del Instituto de Historia del Consejo Superior de Investigaciones Científicas.

Se ha realizado un estudio de la composición elemental mediante Fluorescencia de Rayos X (XRF) empleando un equipo portátil del CNA; metalografía combinada con Microscopio Electrónico de Barrido (SEM) para identificación de compuestos, fases y estructuras metalográficas que permiten estudiar procesos de producción y, finalmente, análisis de isótopos de plomo mediante ICPMS (Espectrometría de masas con fuente de plasma acoplada inductivamente) con el fin de estudiar la posible procedencia del casco a partir de su composición isotópica (HUNT ORTIZ, 2014). Dado que la composición de la aleación química varía entre grupos estilísticos y según sea su procedencia, estos estudios pueden contribuir a acotar el posible origen del casco de Málaga.

En el Laboratorio de Análisis Químicos del IAPH se ha analizado la pieza empleando como métodos de análisis el examen preliminar con microscopio estereoscópico; la observación al microscopio óptico con luz reflejada de la sección transversal de la muestra (metalografía) para analizar la matriz metálica y los productos de corrosión y, por último, el estudio al Microscopio Electrónico de Barrido (SEM) y microanálisis elemental mediante Energía Dispersiva de Rayos X (EDX) de las estratigrafías para estudiar la composición elemental y posibles inclusiones en la matriz metálica. A través de la sección transversal de una lámina metálica mediante la observación al microscopio óptico con luz reflejada se ha estudiado la completa mineralización del metal del casco. Se ha identificado, así mismo, una sucesión de capas de productos de corrosión de óxidos de cobre, carbonatos y cloruros de cobre. (GÓMEZ MORÓN, 2014).

Si bien el estudio analítico no se ha concluido en su totalidad, sí se ha podido determinar que se trata de un bronce binario con bajo contenido en estaño, como es habitual en los cascos griegos, y con hierro como principal impureza. Debido al proceso de mineralización que ha sufrido el metal del casco, se ha transformado en sus minerales. Este hecho ha cambiado radicalmente la apariencia que en su día tuvo este objeto como componente fundamental de la panoplia del guerrero ya que tanto la forma como el brillo le conferirían una gran vistosidad.

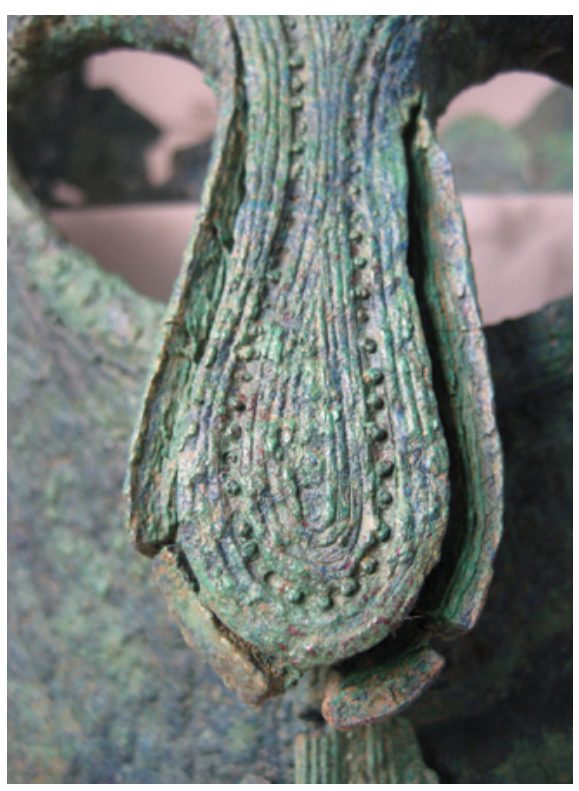

Protector nasal | foto Fondo Gráfico IAPH (Constanza Rodríguez Segovia)

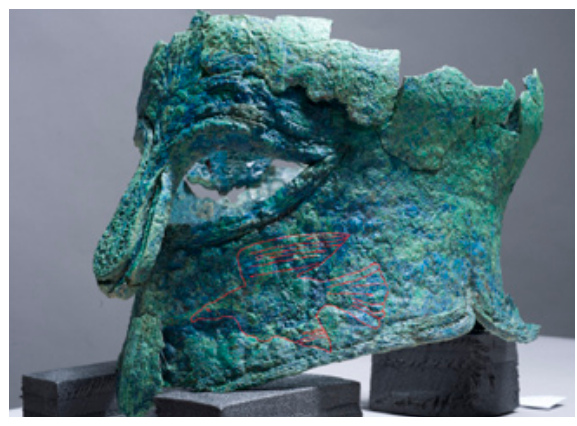

Águila inferior en el cubre-mejillas izquierdo | foto Fondo Gráfico IAPH (Eugenio Fernández); dibujo sobreimpreso Constanza Rodríguez Segovia

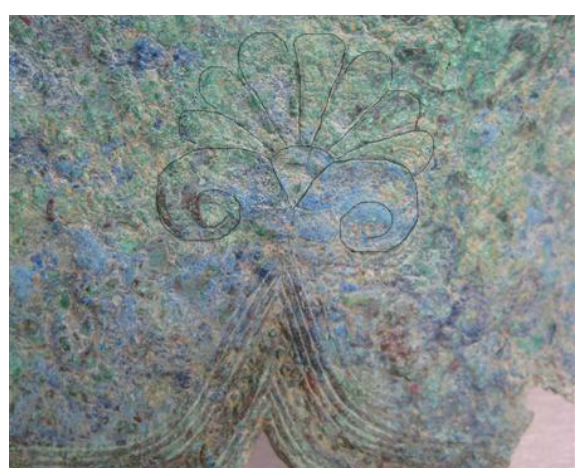

Palmeta de loto en lateral izquierdo | foto Fondo Gráfico IAPH (Constanza Rodríguez Segovia) 


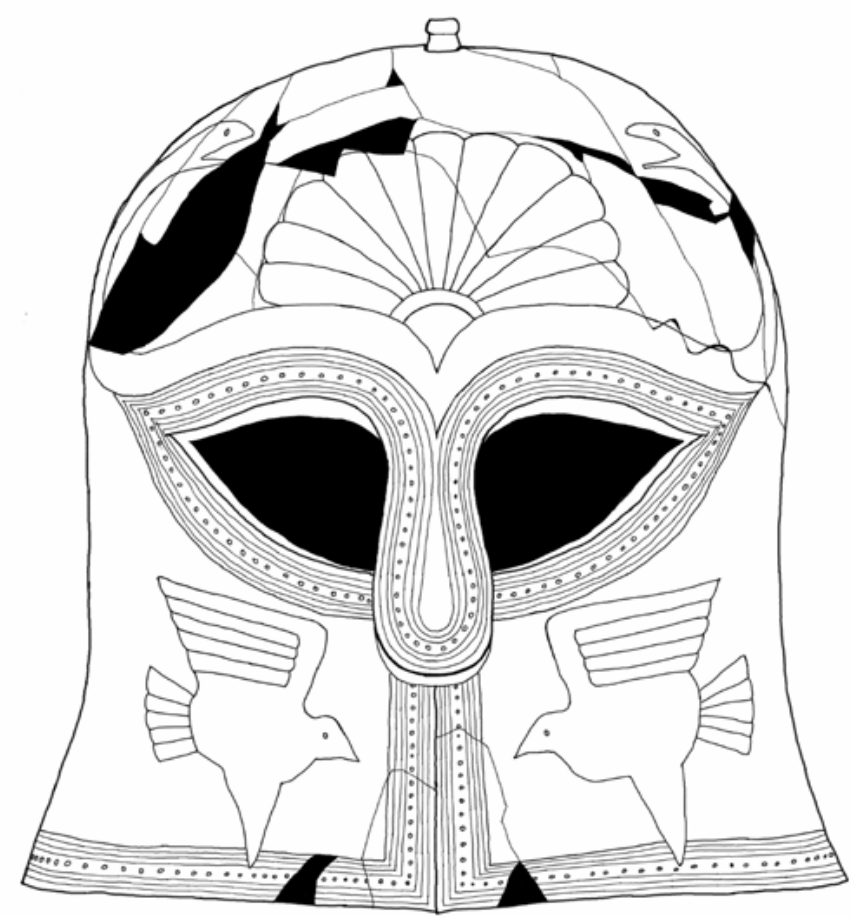

Reconstrucción del frente del casco | dibujo Álvaro Hervás Crespo
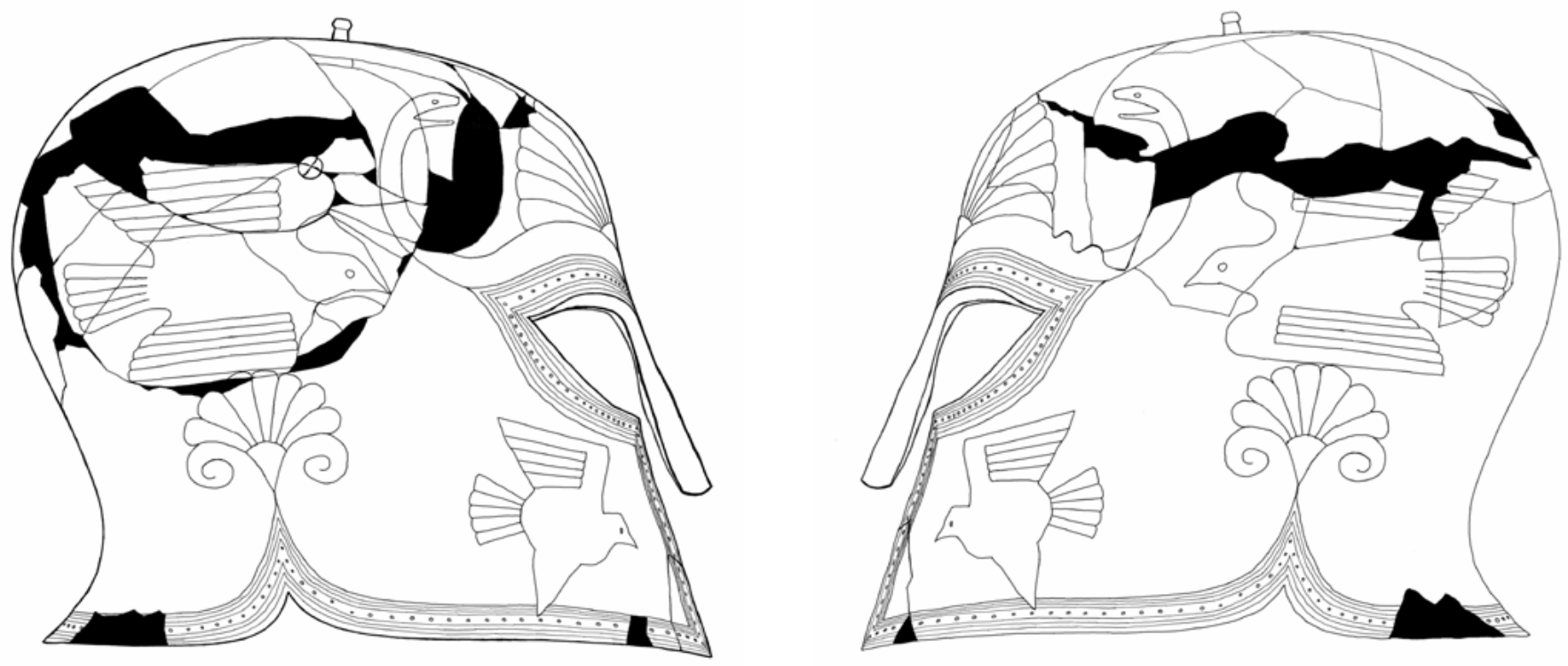

Reconstrucción del lateral derecho e ziquierdo | dibujos Álvaro Hervás Crespo 

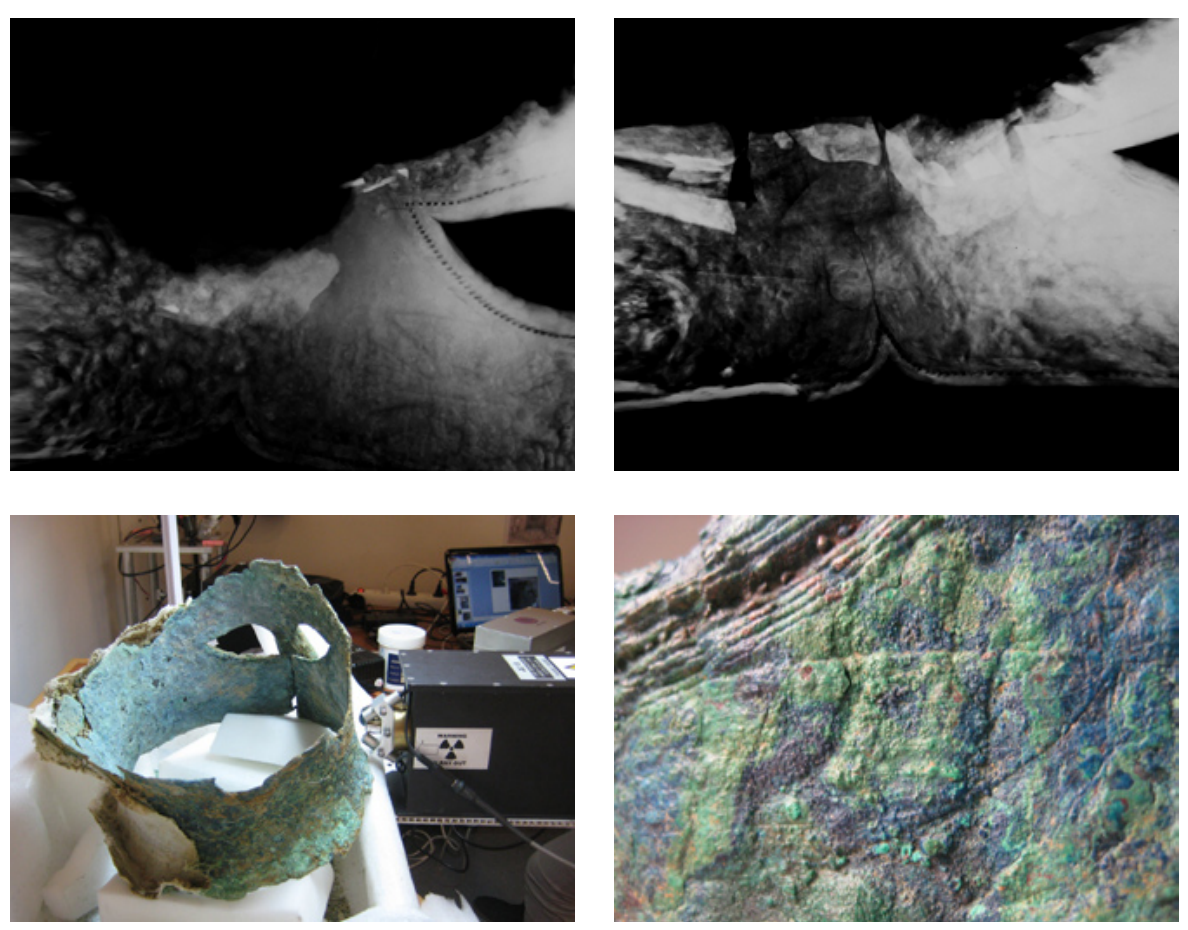

Por lo tanto, el casco ha llegado a nosotros convertido en productos de corrosión de colores verdes, azules y rojos de diferente tonalidad que corresponden fundamentalmente a carbonatos de cobre (malaquita y azurita), cloruros de cobre y, en menor medida, óxido de cobre (cuprita).

Para identificar los restos de una materia marrón adherida a un fragmento de bronce hallado en el interior del casco se proporcionó al Laboratorio de Análisis Biológicos del IAPH una muestra para su análisis. Tras su estudio mediante lupa binocular y microscopio óptico con luz transmitida y reflejada, se observó un aglomerado de materia orgánica amorfa, no siendo posible determinar ninguna estructura biológica debido al estado de deterioro que presentaba.

Tampoco se observó birrefringencia con luz polarizada. Sin embargo, el resultado de las pruebas a las que se sometió la muestra permitió descartar que se tratara de fibras vegetales pues su comportamiento era propio de las fibras animales como el colágeno lo que permite pensar en la posible existencia en su día de un forro de cuero en el interior del casco (MENGUIANO CHAPARRO, 2014).

Por lo que se refiere a las tierras que fueron recogiéndose en bolsas de cierre hermético durante el proceso de vaciado del casco se procedió al cribado de parte de las mismas para tratar de localizar materiales orgánicos empleando
Estudio radiográfico (arriba) | fotos Fondo Gráfico IAPH (Eugenio Fernández Ruiz)

Análisis XRF (abajo izquierda) | foto Fondo Gráfico IAPH (Constanza Rodríguez Segovia)

Pátina en cubre-mejillas izquierdo (ala del águila) (abajo derecha) | foto Fondo Gráfico IAPH (Constanza Rodríguez Segovia) 


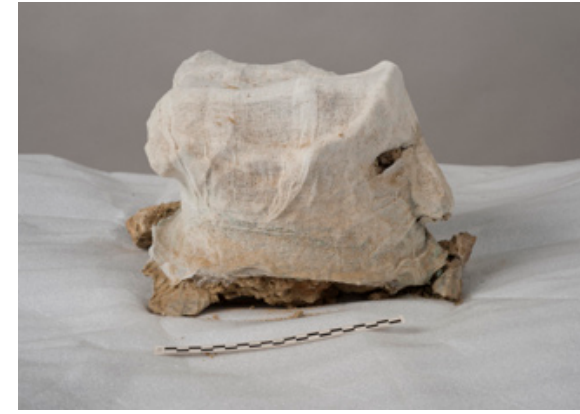

Casco engasado | foto Fondo Gráfico IAPH (Eugenio Fernández Ruiz)
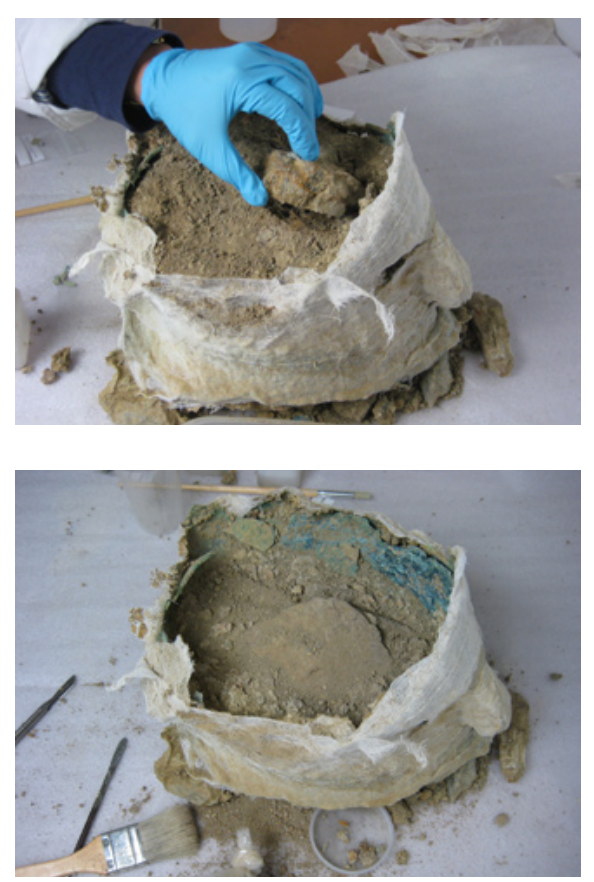

Vaciado del interior | fotos Fondo Gráfico IAPH (Constanza Rodríguez Segovia)

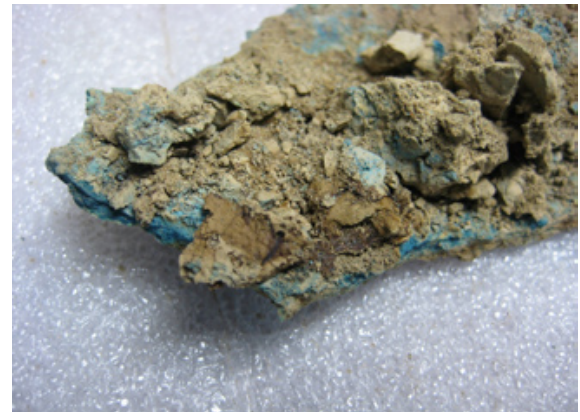

Fragmento con restos de materia orgánica | foto Fondo Gráfico IAPH (Constanza Rodríguez Segovia) dos tamices dispuestos en columna de 4 y $2,5 \mathrm{~mm}$ de luz de malla. Se recuperaron algunos restos orgánicos, mayoritariamente caracoles de pequeño tamaño, así como un un pequeño fragmento de carbón (GAMERO, 2014).

\section{ESTADO DE CONSERVACIÓN Y TRATAMIENTO}

Debido a su fragilidad, el casco se extrajo en bloque y engasado con la tierra que contenía en su interior así como con las piedras sobre las que descansaba.

Para su traslado al IAPH se utilizó una caja metálica que contenía también 27 fragmentos sueltos en bolsa de polietileno con n. ${ }^{\circ}$ de inventario 1146.

En primer lugar, tras desengasar la parte superior, se procedió a realizar el vaciado de las tierras del interior en sucesivas capas o estratos de tal manera que se pudiera tener constancia de la naturaleza del contenido y su ubicación. En el nivel superficial se encontraron 2 caracolillos, pequeños fragmentos de bronce y una piedra. Los fragmentos se introdujeron en una bolsa de polietileno de cierre hermético como el resto de los hallados posteriormente.

Según se fue avanzando se pudieron ver fragmentos de bronce y piedras. Entre la $2 .^{a}$ y $3{ }^{a}$ capa había una piedra de mayor tamaño y fragmentos caídos junto a las paredes internas. En la $4 .^{a}$ capa lo más destacable fue el hallazgo de un gran fragmento de la calota del casco con un remache, así como una piedra de gran tamaño.

Ante la posibilidad de que pudiera conservarse algún resto de materia orgánica de un posible gorro que protegiera la cabeza de su poseedor se observó minuciosamente la tierra y los fragmentos por si se detectaba algún resto de este material. Durante el proceso todo lo que parecía ser de naturaleza distinta a la tierra, de un tono más oscuro y consistencia diferente, se fue guardando para llevar a cabo posteriormente el análisis en el Laboratorio de Biología. Al retirar la piedra se observaron restos de posible materia orgánica tanto en su base como en la tierra.

En la última capa se encontraban las piedras sobre las que descansaba el casco. Su disposición no parecía casual tal como daban a entender las uniones entre las mismas.

Una vez vaciado su interior se continuó con el proceso de desengasado. La gasa se fue desprendiendo con ayuda de un disolvente orgánico. Muchos de los fragmentos de la zona superior se encontraban caídos y mezclados con tierras mientras que otros permanecían unidos al resto de la pieza únicamente por la gasa. Por este motivo fue necesario ir documentando el pro- 
ceso mediante fotografías para poder tener alguna referencia de la ubicación de los fragmentos que se iban desprendiendo al tiempo que se iba dejando constancia también de la ubicación de aquellos que estaban sueltos pero permanecían en su posición. Estos se fueron guardando en bolsas de polietileno de cierre hermético con los datos de su localización para facilitar la posterior tarea de adhesión de fragmentos.

Terminada esta fase se pudo tener una mejor idea del estado de conservación del casco pudiéndose destacar su alto grado de fragmentación, su mineralización y la existencia de numerosas grietas y fisuras. La superficie estaba recubierta por tierras de la excavación consolidadas con la resina acrílica que se aplicó durante el proceso de engasado in situ. Las pátinas correspondían a los típicos tonos verdes y azules de los productos de corrosión del bronce, es decir, carbonatos principalmente (malaquita $\mathrm{Cu}_{2} \mathrm{CO}_{3}(\mathrm{OH})_{2}$ y azurita $\mathrm{Cu}_{3}\left(\mathrm{CO}_{3}\right)_{2}(\mathrm{OH})_{2}$ ), cloruros de cobre (nantokita $\mathrm{CuCl}$ y atacamita $\mathrm{Cu}_{2} \mathrm{Cl}(\mathrm{OH})_{3}$ ) y óxidos de cobre (cuprita $\mathrm{Cu}_{2} \mathrm{O}$ ). En algunos bordes de fractura de los fragmentos se podía observar una estratificación consistente en óxidos de cobre recubiertos de carbonatos mientras que, en la mayoría de los casos, se trataba únicamente de carbonatos.

Uno de los aspectos más reseñables del proceso de corrosión que ha tenido lugar es la existencia de una corrosión en placas de tal forma que en muchas zonas se han conservado fragmentos de cierto grosor en la parte más externa que se han desprendido de una segunda capa de corrosión más fina y poco uniforme frecuentemente constituida sólo por azurita. El proceso de elaboración es, probablemente, el responsable de este tipo de deterioro ya que hay que tener presente que fue elaborado con un bronce laminado al que se le fue dando forma martilleándolo y recociéndolo. El batido ha generado una forma de corrosión concreta en la que destaca la exfoliación del metal.

Hay que mencionar asimismo la diferencia de grosor en los fragmentos que oscilaba entre $3.97 \mathrm{~cm}$ de grosor máximo y 0,02 mm de grosor mínimo según su ubicación en la pieza. En el extremo superior se apreciaban zonas en las que existía un borde de fractura de cierto grosor mientras que en el lateral derecho y en la parte trasera el borde era extremadamente fino indicando que en estas zonas no era posible recuperar ninguna posible unión con los fragmentos hallados en el interior del casco. En el lateral izquierdo, a la altura del parietal, hay una zona del metal rehundida, que ha originado una fractura concéntrica con una deformación evidente hacia el interior que se produciría durante el periodo de uso del casco.

Para eliminar los restos de la resina acrílica utilizada en el engasado in situ se realizó una limpieza química con disolvente orgánico (acetona) de forma que, con la ayuda de una brocha, se fue retirando junto con la tierra adherida a la superficie.
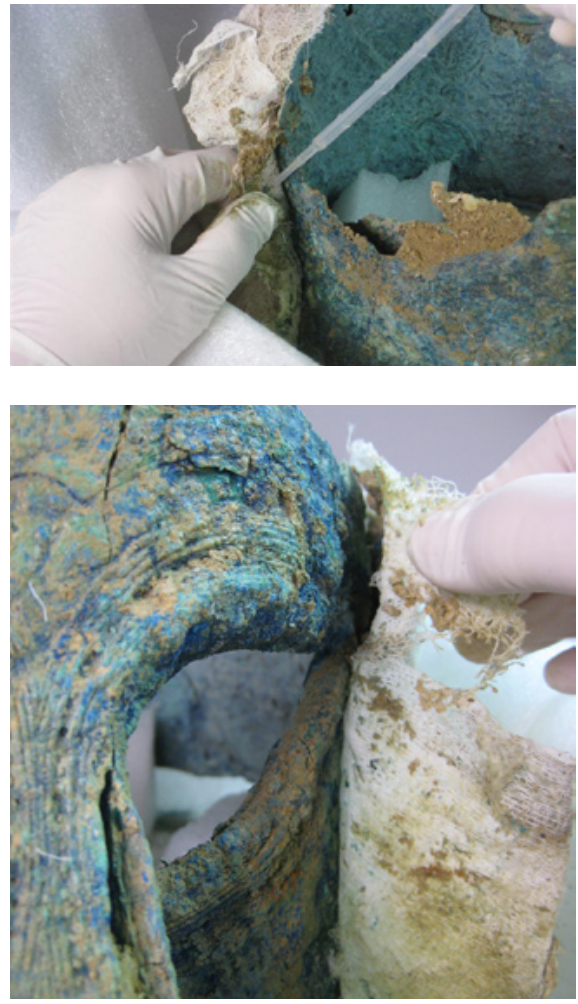

Proceso de desengasado

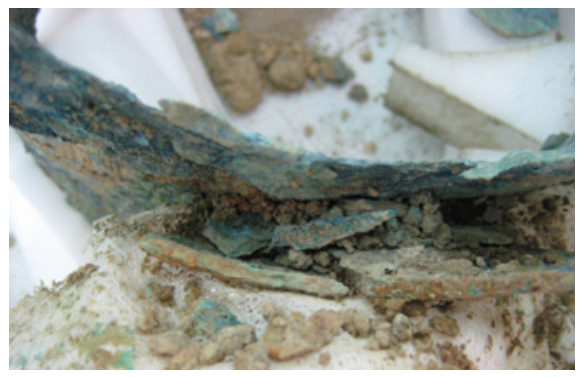

Fragmentos desprendidos entre la gasa y el metal del casco

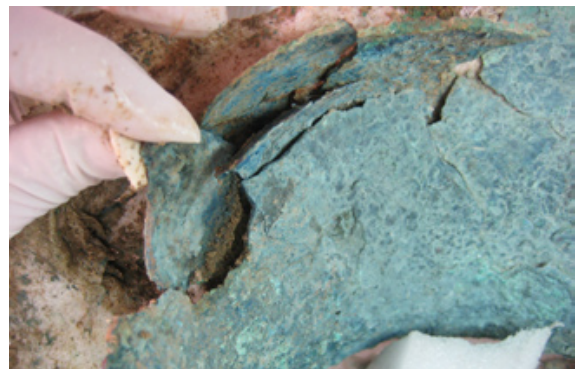

Búsqueda de ubicación de fragmentos | fotos Fondo Gráfico IAPH (Constanza Rodríguez Segovia) 
Proceso de limpieza | fotos Fondo Gráfico IAPH (Constanza Rodríguez Segovia)

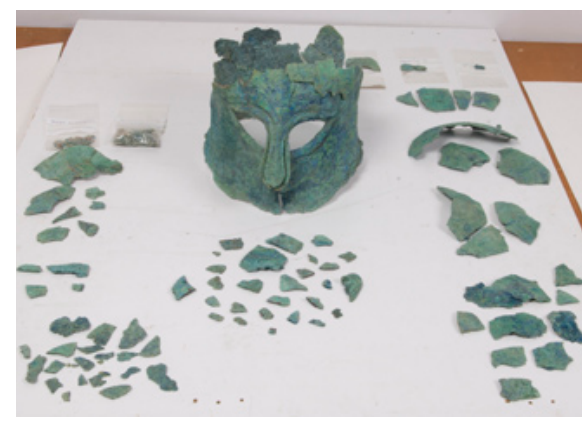

Casco y múltiples fragmentos | foto Fondo Gráfico IAPH (Eugenio Fernández Ruiz)

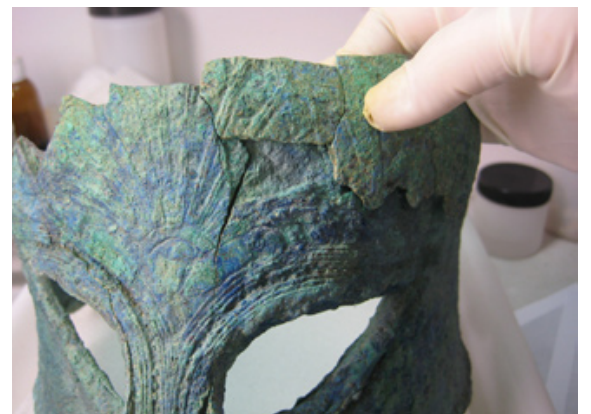

Unión de fragmentos | fotos Fondo Gráfico IAPH (Constanza Rodríguez Segovia)
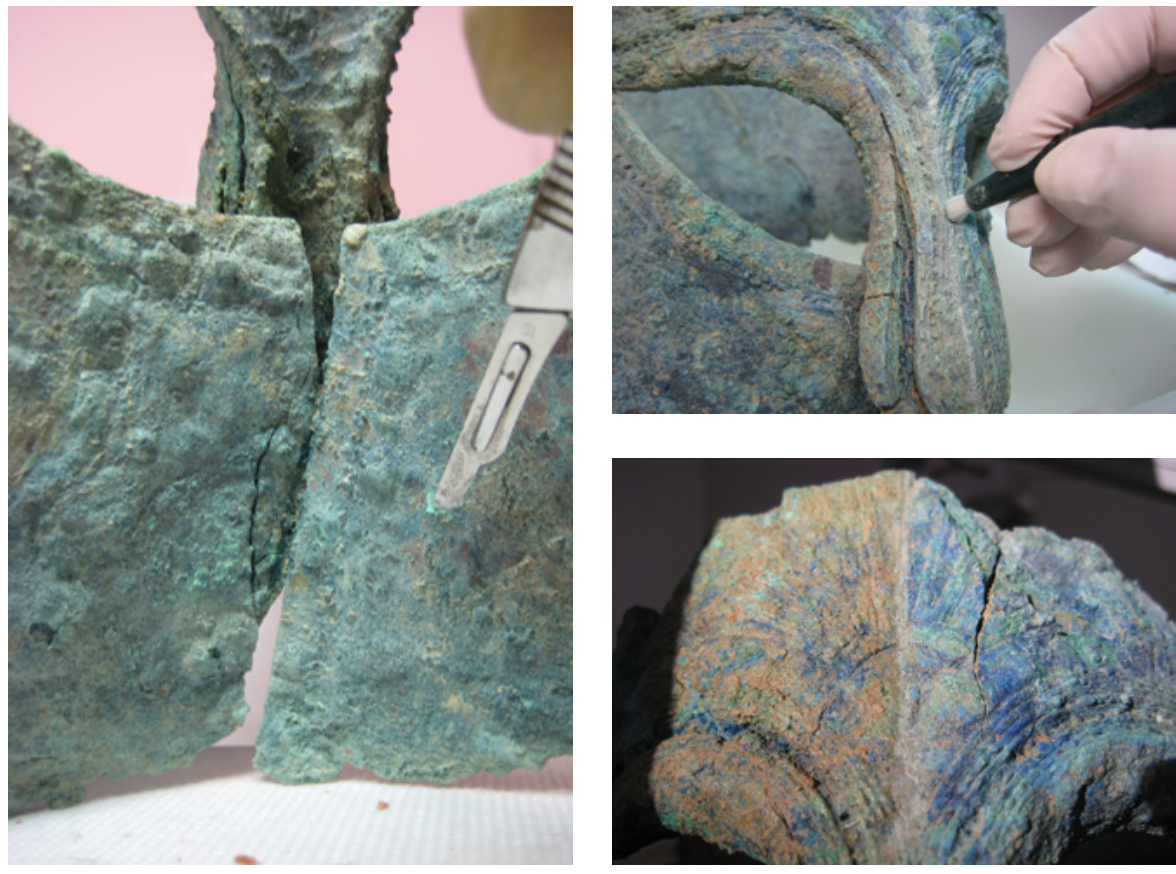

A través de la limpieza mecánica posterior que se llevó a cabo bajo lupa binocular empleando bisturí, microtorno y fibra de vidrio se eliminaron los restos de tierra restantes y productos de corrosión tanto en la superficie externa como en el interior del casco.

Posteriormente se abordó la limpieza de los numerosos fragmentos: Ios hallados durante el vaciado entre la tierra; los que acompañaban al casco pero en una bolsa aparte; y, finalmente, los que se fueron desprendiendo de la gasa por ser ésta su única unión con el resto de la pieza. La limpieza se completó con la introducción de los fragmentos en un baño de alcohol-acetona.

La finalidad de la inhibición que constituyó el siguiente proceso es aislar la pieza de la humedad y el oxígeno mediante la formación de una película estable sobre su superficie para ralentizar la activación del proceso de corrosión. Se efectuó mediante la inmersión tanto del casco como de todos los fragmentos en baño de benzotriazol al 3\% en etanol. Tras el secado en estufa los escasos focos de cloruros activos que pudieron observarse se sellaron con óxido de plata.

La búsqueda de posibles uniones entre los fragmentos fue la tarea más ardua debido a las características que presentaban los bordes de fractura (escaso grosor, deformación) y debido asimismo al tipo de corrosión ya mencionado. De aquellos que se encontraban adheridos a la gasa por la resina acrílica una buena parte se pudieron unir. En total se trata de 34 fragmentos, 

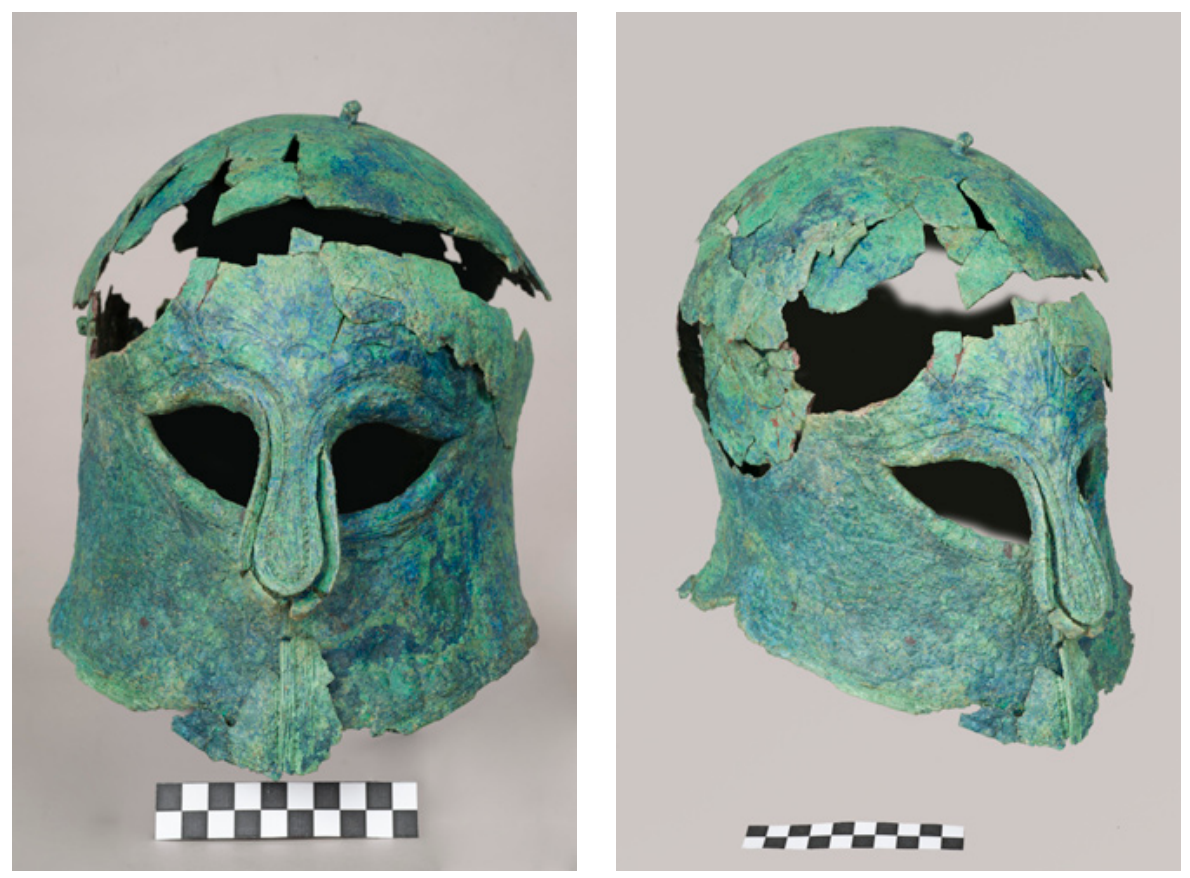

Estado final tras la restauración | fotos Fondo Gráfico IAPH (Eugenio Fernández Ruiz)

algunos con decoración como los de la zona superior del frontal sobre el ojo izquierdo. Al eliminar la tierra durante el vaciado se recuperaron 42 fragmentos, 19 de ellos de pequeño tamaño, además de un remache hallado en la 4. ${ }^{\text {a }}$ capa. En su mayoría corresponden a la calota por lo que esta pudo ser reconstruida parcialmente.

A estos últimos hay que añadir los 27 fragmentos conservados en la bolsa que acompañaba a la pieza que, en algunos casos, tenían decoración.

Para las uniones se empleó un adhesivo tipo cianoacrilato y por el interior se reforzaron a modo de cosido con tiras de papel japonés impregnado en el mismo adhesivo con la idea de aportar una mayor resistencia mecánica al conjunto.

Al superponer la parte de calota reconstruida sobre el resto del casco se puede ver que en el lateral izquierdo las uniones coinciden en muchos casos pero esto no ocurre en el derecho donde los bordes de fractura están muy deteriorados por la corrosión impidiendo posibles uniones. Por este motivo su exposición requiere un montaje ex profeso

En el caso de aquellas uniones en las que la separación entre sus bordes era más acentuada debido a las deformaciones existentes se pusieron puntos de resina epoxy para reforzarlas. Para la aproximación de color se incorporaron a la resina pigmentos al barniz. 


\section{Agradecimientos}

A Leonor Medina Romera,

conservadora-restauradora de bienes culturales (especialidad arqueología) del Museo Arqueológico de Sevilla; y a Álvaro Hervás Crespo, arqueólogo, conservador-restaurador de bienes culturales (especialidad arqueología).
Como último proceso se aplicó una doble película protectora para reforzar el aislamiento frente a los agentes que pueden desencadenar procesos de corrosión. La protección consistió en una primera capa de Incralac (aceite de semilla de soja, etil metacrilato, butil acrilato copolímero (B44), tolueno, etanol y benzotriazol) y una segunda capa de cera microcristalina en White Spirit.

A modo de conclusión hay que mencionar que, si bien la pérdida de fragmentos no permite recuperar la integridad del casco, durante la fase de unión de estos últimos se pudo comprobar la existencia de una mayor cantidad de metal conservado de lo que se pensaba en principio debido a que parte de los mismos se encontraba mezclada con la tierra en el interior de la pieza. Uno de los aspectos más gratificantes fue ir descubriendo detalles de la decoración a lo largo de las distintas fases de las que ha constado la intervención.

\section{FICHA TÉCNICA. ESTUDIO Y RESTAURACIÓN DE UN CASCO GRIEGO DEL MUSEO DE MÁLAGA}

\section{Conservación-restauración:}

Constanza Rodríguez Segovia, Área de Tratamiento, Centro de Intervención del Patrimonio Histórico, IAPH

Estudio histórico-arqueológico:

Esther Núñez Pariente de León, Departamento de Estudios Históricos y Arqueológicos, Centro de Intervención, IAPH

Estudio analítico:

Auxiliadora Gómez Morón, Laboratorio de Análisis Químicos, Centro de Inmuebles, Obras e Infraestructuras, IAPH

Victor Menguiano Chaparro, Laboratorio de Análisis Biológicos, Centro de Inmuebles, Obras e Infraestructuras, IAPH

Miguel Gamero Esteban, Laboratorio de Paleobiología, IAPH

Mark. A. Hunt Ortiz, arqueólogo, especialista en arqueometría

Miguel Angel Respaldiza, Inés Ortega Feliú, Simona Scrivano, Centro Nacional de Aceleradores

Carolina Gutiérrez Neira, Laboratorio de Arqueometría de Materiales (LAM), Instituto de Historia del Consejo Superior de Investigaciones Científicas (Madrid)

Estudio fotográfico y radiográfico:

Eugenio Fernández Ruiz, Laboratorio de Medios Físicos de Examen, Centro de Intervención, IAPH 


\section{BIBLIOGRAFÍA}

- AlBelda AlBeRT, J.; OBERMAiER, H. (1988) El casco griego de Huelva. Huelva: Diputación Provincial, 1988 (Clásicos de la arqueología de Huelva; 1/1988) Reprod. facs. de la separata de: Boletín de la Academia de la historia, t. 98, cuaderno 2 (1931), pp. 642-648. Contiene además: Un casco griego de España / por el Dr. Adolf Schulten. Un casco griego de Huelva / Ricardo Olmos Romera

- BOTTINI, A.; EGG. M.; VON HASE, F. W. et ál. (1988) Antike Helme. Handbuch mit Katalog. Sammlung Lipperheide und andere Bestände des Antikenmuseums Berlin. Mainz: Römisch-Germanischen Zentralmuseums, 1988 (Monographien Des Römisch-Germanischen Zentralmuseums, 14)

- GABALDÓN MARTíNEZ, M. ${ }^{a}$ M. (2004) Ritos de armas en la antigüedad. Gladius, anejos 7, 2004, p. 122

- GABALDÓN MARTínEZ, M. ${ }^{a}$ M. (2010) Sacra loca y armamento. Algunas reflexiones en torno a la presencia de armas no funcionales en contextos rituales. Gladius, 30, 2010, pp. $191-212$

- GAMERO, M. (2014) Recuperación y conservación de materiales de origen orgánico de los nueve bienes arqueológicos del museo de Málaga. Informe de trabajo, 2014

- GARCíA Y BELLIDO, A. (1948) Hispania Graeca. Barcelona: [s.n.], 1948 (Imp. Casa P. de Caridad), pp. 82 y ss.

- GÓMEZ MORÓN, A. (2014) Caracterización de materiales metálicos de la encomienda de nueve bienes muebles del museo de Málaga. Informe de trabajo, 2014

- GONZÁlEZ RODRÍGUEZ, R.; BARRIONUEVO CONTRERAS, F.; AGUILAR MOYA, L. (1997) Notas sobre el mundo funerario de la Baja Andalucía. En Actas de las Jornadas La Andalucía Ibero-Turdetana (siglos VI-IV a.C.). Huelva Arqueológica XIV, 1997, pp. 247-268

- GRAELlS I FABREgAT, R. (2013) Die Helme von Olympia: Ein Beitrag zu Waffenweihungen in griechischen Heiligtümern by Heide Frielinghaus. American Journal of Archaeology, 117, 2, 2013

- GRAELlS I FABREgAT, R. (2014) Mistophoroi ex Iberias. Una aproximación al mercenariado hispano a partir de las evidencias arqueológicas (s. VI - IV a.C.). Venosa: Osanna Edizioni, 2014

- HANSON, V. D. (1989) The western. Way of war. Infanty Battle in Classical Greece. Los Ángeles: University California Press, 1989

- HUGHES, R. (1993) Artificial patination, in Metal Plating and Patination. Oxford: Butterworth-Heinemann, 1993

- HUNT ORTIZ, M. (2014) Informe Arqueometalúrgico.
Metales. Museo Málaga. Informe de trabajo, 2014

- JIMÉNEZ ÁVILA, J. (2002) La toréutica orientalizante en la Península Ibérica. Madrid: Real Academia de la Historia, 2002 (Bibliotheca archaeologica hispana, 16. Studia hispano-phoenicia, 2)

- MANTI, P.; WATKINSON, D. (2008) From Homer to Hoplite: Scientific Investigations of Greek Copper Alloy Helmets. Science and Technology in Homeric Epics. History of Mechanism and Machine Science, Vol. 6, 2008, pp. 167179

- MANTI, P. (2012) Shiny helmets: investigation of tinning, manufacture and corrosion of Greek helmets (7th-5th c. BC). Tesis PhD. Department of Archaeology and Conservation. School of History, Archaeology and Religious Studies. Cardiff University, 2012

- MENGUIANO CHAPARRO, V. (2014) Análisis biológico Casco Guerrero A/DJ14422/1.3.76.5 Actividad arqueológica Jinete-Refino. Informe de trabajo, 2014

- MONTERo VítoRES, J. (2002) El yacimiento de Dehesa de Bolaños en el marco de la Bahía de Cádiz. De Portus Menesthei a Portus Gaditanus. Revista de Historia de Jerez, 8, 2002, pp. 35-66

- QUESADA SANZ, F.; VALERO TÉVAR, M. A. (20112012) Un casco variante del grupo italo-calcídico en la necrópolis de los Canónigos, Arcas del Villar (Cuenca). Cuadernos de Prehistoria y Arqueología Universidad Autónoma de Madrid, n. ${ }^{\circ}$ 37-38, pp. 2011-2012

- OLMOS, R. (1992) Iconografía y culto a las aguas de época prerromana en los mundos colonial e ibérico. Espacio, Tiempo y Forma [en línea], Serie II, $\mathrm{H}^{\mathrm{a}}$ Antigua, t. V, 1992, pp. 103-120 <http://revistas.uned.es/index.php/ ETFII/article/view/4187/4026> [Consulta: 24/06/2015]

- SCHULTEN, A. (1979) Tartessos. 2. a edición. Madrid: Espasa-Calpe, 1979, p. 82 (Colección Austral, 1471)

- TIEMBlo MAGRO, A. (1994) Un nuevo casco griego de bronce: posiblemente hallado en España. Revista de Arqueología, 158, 1994, pp. 32-35

- TREISTER, M. Y. (1996) The Role of Metals in Ancient Greek History. Leiden: E.J. Brill, 1996 